\title{
CULTURAL PROBLEMS OF TURKISH STUDENTS WHILE LEARNING ENGLISH AS A FOREIGN LANGUAGE
}

\author{
Binnur Genç İlter \\ Cem Oktay Güzeller
}

\begin{abstract}
Teaching a foreign language by means of culture has become increasingly widespread nowadays. As an English teacher, we should try to keep students interested in the classroom. Culture is one of the best ways of motivation and it also provides students with useful models of authentic use of the language in the classroom. On the other hand, cultural differences may bring some problems into the classrooms while learning a foreign language.

This article has attempted to help teachers understand and solve their students' cultural problems in the classroom. It also aims at examining the students' ideas about the cultural differences.
\end{abstract}

Keywords: Culture, cultural values, ELT, cultural problems

\section{INGILIZCEYYI YABANCI DIL OLARAK ÖĞRENEN TÜRK ÖĞRENCILERIN KARŞILAŞTIKLARI KÜLTÜREL SORUNLAR}

$\ddot{O}_{z e t}$

Son zamanlarda yabancı bir dili kültürle birlikte ögrretmek giderek yaygınlaşmaya başladı. İnilizce öğretmeni olarak bizler sınıfta öğrencilerin ilgisini sürekli kılmă̆a çalışmalıyız. Kültür öğrenciyi güdülemenin en iyi yollarından biridir,öğrencilerin dili daha iyi algllayabilmeleri için otantik ortamlar oluşturur. Yabancı bir dili öğrenirken ortaya çıkan kültürel faklılıklar da bazı sorunlara neden olabilir.

Bu makalede Türk öğrencilerinin karşılaştıkları sorunlar ele alınmakta ve ögretmenlere bu sorunların aşılması için bazı önerilerde bulunulmaktadır.

Anahtar sözcükler: Kültür, kültürel değgerler, Ingilizce ögretimi, kültürel problemler 


\section{Introduction}

It is a fact that the term 'culture' contains beliefs, ideas, values and knowledge. According to Adaskou et al 1990, culture is composed of literature, music, fine arts, films and plays. Culture also shows the life style in the target community.

Culture and teaching language cannot be separated, thus culture must be taught while teaching a target language. If languages are taught without their cultures, students are only the strangers who aren't familiar with the target languages. It is important to mention that cultural elements of the target language should be taught carefully.

Widdowson 1990 claims that a child acquires the semantic and syntactic systems together with his cultural knowledge, on the other hand, while learning a foreign language the student tends to acquire the language code of another community. The idea that a child acquires his native language together with its culture indicates that language can not be taught without cultural background.

Ellis 1990 states that students who are interested in the social and cultural life of the native country can learn a foreign language better than the other students, therefore students should be given support which will enable them to acquire the necessary cultural knowledge.

Jiang 2000 implies that it is impossible to teach the target language without teaching the target culture. The students can be exposed to an empty frame of language if they don't learn cultural aspects of the target country.

According to Chastain 1988 teaching culture in EFL classrooms has got a crucial role of the course. It is a well-known fact that language teaching and culture are bound and during international communications students require social and cultural awareness.

\section{Importance of the study}

Teaching culture in EFL classrooms makes communication successful for the students who want to learn the target language in natural ways.

The use of target culture elements in EFL classrooms encourages the students to learn English effectively. This paper shows the relationship between culture and language learning.

\section{Purpose of the study}

This study aims at researching arguments of culture, teaching culture and cultural problems of Turkish students while learning English. The purpose of this article is also to give an idea about the problems based on cultural differences in the classroom. 


\section{Problem of the study}

In this study, the following research questions were treated:

1. How can we select the proper cultural materials for our students?

2. What is the attitude of Turkish students towards the cultural elements of EFL lessons?

3. What do the students need as cultural elements in EFL classrooms?

\section{Method of the study}

In this research paper a survey was designed to understand the students' point of view about language and culture. The survey was divided into two sections (Appendix 1). The first section was about the personal information of the students and the second section was about the attitude of the students towards the cultural elements of textbooks in EFL classrooms. The questionnaire was completed by 150 preparatory class students at Akdeniz University in Antalya. At Akdeniz University, the students are required to learn English and reach upper intermediate level by the end of the year. The questionnaire was administered to the students with the help of their teachers. The data analysis was completed but they didn't aim at carrying out in depth statistical evolution of data.

\section{Limitations of the study}

This study is limited to Akdeniz University which provides one year preparatory class education. This study is also limited to 150 students of all the departments.

\section{Findings}

\subsection{Findings based on the students' general information}

- $79.2 \%$ of the students were between the ages of $17-21 ; 17.9 \%$ of them were between $21-24$ and $2.8 \%$ of them were 25 or over it.

- $30.2 \%$ of the students were girls and $69.9 \%$ of the students were boys.

- In the research, $71.7 \%$ of the students were from state high schools, $16 \%$ from Anatolian high schools, 3.8\% from super high schools, and 3.8\% from private high schools. $3.8 \%$ of them were from science high schools, only $0.9 \%$ of them from technical high schools.

- The students were from seven different regions all over Turkey.

- $20.8 \%$ of the respondents were from Marmara, 10.4\% from Ege, 34.9\% from Akdeniz, 13.2\% from Middle Anatolia, 5.7\% from East Anatolia, 5.7\% from

South - East Anatolia and $9.4 \%$ of them were from Karadeniz regions (Appendix 2). 


\subsection{Findings based on the students' ideas about culture}

- $62 \%$ of them stated that they wanted to learn the culture of the target language they are learning.

- $41,5 \%$ of the students said that they wanted to learn cultural elements of the target language only from reading passages.

- $10.4 \%$ of them stated that they wanted to learn it just like their cultural values.

- $42.5 \%$ of them declared that they wanted to learn it in order to understand the target language better.

- $58.5 \%$ of the students implied that they didn't like and understand the cultural words which belong to the target language.

- $70.8 \%$ of the students mentioned that learning different cultures provided them with different point of view about the world in which they lived.

- $29.2 \%$ of them indicated that different cultural values developed their critical thought.

- $81.1 \%$ of the students stated that they would not lose their national identity while learning cultural values that belong to another country.

- $38.7 \%$ of the students thought that the course books are aimed at teaching En-

glish culture completely.

- $24.5 \%$ of them stated that English course books didn't give importance to

Turkish culture.

- $36.8 \%$ of them thought that publishers only wanted to teach cultures of the countries where English was spoken.

- All the students over 25 explained that unknown words belonging to the target language's culture bothered them. It shows that the older the students are less tolerant about different cultures.

- $78.9 \%$ of the students between 21 - 24 and all the students over 25 claimed that learning different cultures provided them with an idea of comparison between their culture and the other culture.

- According to the results, the students from super high schools and technical high schools wanted to learn British/American culture more than those of Anatolian, state, private and science high schools.

- All the students from super high schools and technical high schools mentioned that they could get a different view about the world by comparing their culture with target language's culture.

- $75 \%$ of the students from science schools stated that they wanted to learn the culture in order to understand the target language. 
- If we have a look at the gender, it can be seen that girls with the ratio $68.8 \%$ wanted to learn British/American culture more than the boys.

- The majority of the boys and girls wanted to learn the target language's culture in order to understand the language better

- $59.4 \%$ of the girls mentioned that the unknown cultural words bothered them.

- $68.8 \%$ of the girls wanted to learn British/American culture whereas $54.1 \%$ of the boys wanted to learn these cultures. It shows that girls are more eager to

learn different cultures than boys.

- $71.6 \%$ of the boys explained that different cultures would provide them to gain various thoughts about the world.

- $21.9 \%$ of them mentioned that the course books didn't give importance to Turkish people.

- $37.8 \%$ of the boys thought that publishers tried to teach only their culture.

\section{Conclusion and suggestion}

In conclusion, it can be inferred that the students from different high schools in Turkey have a tendency of learning different cultures by learning the target language. However, they believe that the course books used in EFL classrooms aim at teaching only the target language's culture. The result appears to be very important because it shows that the students wanted to see some cultural values of Turkish people in their course books. Turkish students are conscious enough of the language teaching methods and they know that culture and language learning cannot be separated. Hence, English cannot be taught without British/American culture. The problem is how to select the correct cultural context. The context used in EFL classrooms should be familiar with the students.

It can be suggested that instead of teaching culture of the target language completely, it is more useful to take a cross - cultural approach to English language teaching. By focusing the cross - cultural values, the students will become more conscious to the world's cultural beliefs. It is clear that the students will show tolerance to the universe which they live in when they learn different values of various countries.

\section{References}

Adeskou, K., D. Britten and B. Fashi 1990. Design Decisions on the Cultural Content of a Secondary English Course for Morocco. ELT Journal, 44/1:3-10

Chastain, K. 1988. Developing Second - Language Skills. Theory and Practice. Harcourt Brace Javanov inch: The USA

Ellis, R. 1990. Understanding Second Language Acquisition. OUP: Oxford

Jiang, W. 2000. The Relationship between Culture and Language. ELT Journal 54 / 4: 328-334

Widdowson, H. G. 1990. Aspect of Language Teaching. Oxford: OUP 


\section{A P P E N D I C E S}

\section{Appendix I \\ Part I}

\section{General Information}

1. Your Age:

2. Your Gender:

3. Your School Type:

4. Where did you finish your high school?

\section{Part II}

\section{Students' Ideas About Target Languages' Culture}

1. While learning English, Do you want to learn English / American culture ?
a) Yes
b) No
c) Sometimes

2. If you want to learn English / American culture, how do you want to learn it ?

a) Only in the reading passages about the target culture.

b) Just like my own cultural values.

c) A little bit to understand the target language.

3. Do the words which you don't understand about target language's culture bother you?
a) Yes
b) No
c) Sometimes

4. What kind of contribitutions will you get by learning different cultural values ?

a) It gives us different point of view abut the world we live in and life

b) It develops my critical thoughts.

5. While learning different culture, do you think you will lose your national identity?
a) Yes
b) No
c) Sometimes

6. What kind of cultural problems do you usually face while learning a foreign language?
a) British - American coursebooks wanted to teach only their culture
b) They don't give importance to Turkish culture
c) They only try to teach English spoken countries cultural elements 


\section{Appendix II:}

\begin{tabular}{|l|l|l||}
\hline \hline & Frequency & Percent \\
\hline Girl & 32 & 30,2 \\
\hline Boy & 74 & 69,8 \\
\hline \hline Total & $\mathbf{1 0 6}$ & $\mathbf{1 0 0 , 0}$ \\
\hline
\end{tabular}

Table 1- A) Students' Gender

\begin{tabular}{|l|l|l||}
\hline & Frequency & Percent \\
\hline $\mathbf{1 7}-\mathbf{2 0}$ & 84 & 79,2 \\
\hline $\mathbf{2 1}-\mathbf{2 4}$ & 19 & 17,9 \\
\hline $\mathbf{2 5}-$ & 3 & 2,8 \\
\hline \hline Total & $\mathbf{1 0 6}$ & $\mathbf{1 0 0 , 0}$ \\
\hline
\end{tabular}

Table 1- B) Students' Ages

\begin{tabular}{|l|l|l||}
\hline & Frequency & Percent \\
\hline State & 76 & 71,7 \\
\hline Private & 7 & 3,8 \\
\hline Super & 4 & 3,8 \\
\hline Anatolian and & 1 & 16,0 \\
\hline $\begin{array}{l}\text { Technical } \\
\text { Vacational }\end{array}$ & 0,9 \\
\hline Science & 4 & 3,8 \\
\hline \hline Total & $\mathbf{1 0 6}$ & $\mathbf{1 0 0 , 0}$ \\
\hline
\end{tabular}

Table 1- C) Students' high school types

\begin{tabular}{||l|l|l||}
\hline & Frequency & Percent \\
\hline Marmara & 22 & 20,8 \\
\hline Ege & 11 & 10,4 \\
\hline Akdeniz & 37 & 34,9 \\
\hline İç Anadolu & 14 & 13,2 \\
\hline Doğu Anadolu & 6 & 5,7 \\
\hline Güneydoğu Anadolu & 6 & 5,7 \\
\hline Karadeniz & 10 & 9,4 \\
\hline \hline Total & $\mathbf{1 0 6}$ & $\mathbf{1 0 0 , 0}$ \\
\hline \hline
\end{tabular}

Table 1-D) The regions

\begin{tabular}{|l|l|l|}
\hline & Frequency & Percent \\
\hline Yes & 62 & 58,5 \\
\hline No & 44 & 41,5 \\
\hline \hline Total & $\mathbf{1 0 6}$ & $\mathbf{1 0 0 , 0}$ \\
\hline
\end{tabular}

Table 2: Question 1

\begin{tabular}{||l|l|l||}
\hline & Frequency & Percent \\
\hline $\boldsymbol{A}$ & 6 & 10,0 \\
\hline B & 11 & 18,0 \\
\hline C & 45 & 72,0 \\
\hline \hline Total & $\mathbf{6 2}$ & $\mathbf{1 0 0 , 0}$ \\
\hline
\end{tabular}

Table 3: Question 2

\begin{tabular}{|l|l|l|}
\hline & Frequency & Percent \\
\hline Yes & 62 & 58,5 \\
\hline No & 9 & 8,5 \\
\hline Sometimes & 35 & 33,0 \\
\hline \hline Total & $\mathbf{1 0 6}$ & $\mathbf{1 0 0 , 0}$ \\
\hline
\end{tabular}

Table :4 Question 3

\begin{tabular}{|l|l|l||}
\hline & Frequency & Percent \\
\hline $\mathbf{1}$ & 75 & 70,8 \\
\hline $\mathbf{2}$ & 31 & 29,2 \\
\hline \hline Total & $\mathbf{1 0 6}$ & $\mathbf{1 0 0 , 0}$ \\
\hline
\end{tabular}

Table 5: Question 4

\begin{tabular}{||l|l|l||}
\hline & Frequency & Percent \\
\hline Yes & 20 & 18,9 \\
\hline No & 86 & 81,1 \\
\hline \hline Total & $\mathbf{1 0 6}$ & $\mathbf{1 0 0 , 0}$ \\
\hline
\end{tabular}

Table 6: Question 5

\begin{tabular}{|l|l|l|}
\hline & Frequency & Percent \\
\hline A & 41 & 38,7 \\
\hline B & 26 & 24,5 \\
\hline C & 39 & 36,8 \\
\hline \hline Total & 106 & 100,0 \\
\hline \hline
\end{tabular}

Table 7 : Question 6

\begin{tabular}{|l|l|l|l|l|}
\hline & & Yes & No & Total \\
\hline \multirow{2}{*}{$\mathbf{1 7}-\mathbf{2 0}$} & Frequency & 48 & 36 & 84 \\
\cline { 2 - 5 } & Percent & 57,1 & 42,9 & 100,0 \\
\hline \multirow{2}{*}{$21-\mathbf{2 4}$} & Frequency & 13 & 6 & 19 \\
\cline { 2 - 5 } & Percent & 68,4 & 31,6 & 100,0 \\
\hline \multirow{2}{*}{$25-$} & Frequency & 1 & 2 & 3 \\
\cline { 2 - 5 } & Percent & 33,3 & 66,7 & 100,0 \\
\hline \hline \multirow{2}{*}{ Total } & Frequency & 62 & 44 & 106 \\
\cline { 2 - 5 } & Percent & 58,5 & 41,5 & 100,0 \\
\hline
\end{tabular}

Table 8: The Relations between age and question 1 


\begin{tabular}{||l|l|l|l|l||}
\hline & $\mathbf{A}$ & $\mathbf{B}$ & $\mathbf{C}$ & Total \\
\hline \multirow{2}{*}{$\mathbf{1 7}-\mathbf{2 0}$} & 5 & 7 & 36 & 48 \\
\cline { 2 - 5 } & 10,0 & 15,0 & 75,0 & 100,0 \\
\hline \hline \multirow{2}{*}{$\mathbf{2 1}-\mathbf{4}$} & 1 & 4 & 8 & 13 \\
\cline { 2 - 5 } & 8,0 & 31,0 & 61,0 & 100,0 \\
\hline \hline \multirow{2}{*}{$\mathbf{2 5}-$} & 0 & 0 & 1 & 1 \\
\cline { 2 - 5 } & 0,0 & 0,0 & 100,0 & 100,0 \\
\hline \hline \multirow{2}{*}{ Total } & 6 & 11 & 45 & 62 \\
\cline { 2 - 5 } & 10,0 & 18,0 & 72,0 & 100,0 \\
\hline
\end{tabular}

Table 9: The relations between age and question 2

\begin{tabular}{||l|l|l|l|l||}
\hline & Sometimes & Yes & No & Total \\
\hline \multirow{2}{*}{$\mathbf{1 7}-\mathbf{2 0}$} & 29 & 47 & 8 & 84 \\
\cline { 2 - 5 } & 34,5 & 56,0 & 9,5 & 100,0 \\
\hline \hline \multirow{2}{*}{$\mathbf{2 1}-\mathbf{2 4}$} & 6 & 12 & 1 & 19 \\
\cline { 2 - 5 } & 31,6 & 63,2 & 5,3 & 100,0 \\
\hline \hline \multirow{2}{*}{$\mathbf{2 5}-$} & 0 & 3 & 0 & 3 \\
\cline { 2 - 5 } & 0,0 & 100,0 & 0,0 & 100,0 \\
\hline \hline \multirow{2}{*}{ Total } & 35 & 62 & 9 & 106 \\
\cline { 2 - 5 } & 33,0 & 58,5 & 8,5 & 100,0 \\
\hline
\end{tabular}

Table 10: The relations between age and question 3

\begin{tabular}{||l|l|l|l|}
\hline & A & B & Total \\
\hline \multirow{2}{*}{$\mathbf{1 7}-\mathbf{2 0}$} & 57 & 27 & 84 \\
\cline { 2 - 4 } & 67,9 & 32,1 & 100,0 \\
\hline \hline \multirow{2}{*}{$21-24$} & 15 & 4 & 19 \\
\cline { 2 - 4 } & 78,9 & 21,1 & 100,0 \\
\hline \hline \multirow{2}{*}{$\mathbf{2 5 -}$} & 3 & 0 & 3 \\
\cline { 2 - 4 } & 100,0 & 0 & 100,0 \\
\hline \hline \multirow{2}{*}{ Total } & 75 & 31 & 106 \\
\cline { 2 - 4 } & 70,8 & 29,2 & 100,0 \\
\hline
\end{tabular}

Table 11: The relations between age and question 4

\begin{tabular}{||l|l|l|l||}
\hline & Yes & No & Total \\
\hline \multirow{2}{*}{$17-\mathbf{2 0}$} & 15 & 69 & 84 \\
\cline { 2 - 4 } & 17,9 & 82,1 & 100,0 \\
\hline \hline \multirow{2}{*}{$\mathbf{1}-\mathbf{2 4}$} & 3 & 16 & 19 \\
\cline { 2 - 4 } & 15,8 & 84,2 & 100,0 \\
\hline \hline \multirow{2}{*}{$\mathbf{5}-$} & 2 & 1 & 3 \\
\cline { 2 - 4 } & 66,7 & 33,3 & 100,0 \\
\hline \hline \multirow{2}{*}{ Total } & 20 & 86 & 106 \\
\cline { 2 - 4 } & 18,9 & 81,1 & 100,0 \\
\hline \hline
\end{tabular}

Table12: The relations

between age and question 5

\begin{tabular}{||l|l|l|l|l||}
\hline & A & B & C & Total \\
\hline \multirow{2}{*}{$\mathbf{1 7}-\mathbf{2 0}$} & 33 & 20 & 31 & 84 \\
\cline { 2 - 5 } & 39,3 & 23,8 & 36,9 & 100,0 \\
\hline \hline \multirow{2}{*}{$2 \mathbf{- 2 4}$} & 6 & 5 & 8 & 19 \\
\cline { 2 - 5 } & 31,6 & 26,3 & 42,1 & 100,0 \\
\hline \hline \multirow{2}{*}{$\mathbf{2 5}-$} & 2 & 1 & 0 & 3 \\
\cline { 2 - 5 } & 66,7 & 33,3 & 0,0 & 100,0 \\
\hline \hline \multirow{2}{*}{ Total } & 41 & 26 & 9 & 106 \\
\cline { 2 - 5 } & 38,7 & 24,5 & 36,8 & 100,0 \\
\hline
\end{tabular}

Table13: The relations between age and questions 6

\begin{tabular}{||l|l|l|l|l|}
\hline & & Yes & No & Total \\
\hline \multirow{3}{*}{ State } & Frequency & 43 & 33 & 76 \\
\cline { 2 - 5 } & Percent & 56,6 & 43,4 & 100 \\
\hline \multirow{2}{*}{ Private } & Frequency & 2 & 2 & 4 \\
\cline { 2 - 5 } & Percent & 50,0 & 50,0 & 100,0 \\
\hline \multirow{2}{*}{ Anatolian } & Frequency & 4 & 0 & 4 \\
\cline { 2 - 5 } & Percent & 100,0 & 0,0 & 100,0 \\
\hline \multirow{2}{*}{ Vechnical acational } & Frequency & 11 & 6 & 17 \\
\hline \multirow{2}{*}{ Science } & Percent & 64,7 & 35,3 & 100,0 \\
\hline \hline \multirow{2}{*}{ Total } & Frequency & 1 & 0 & 1 \\
\cline { 2 - 5 } & Percent & 100,0 & 0,0 & 100,0 \\
\cline { 2 - 5 } & Prequency & 1 & 3 & 4 \\
\cline { 2 - 5 } & Frequency & 62 & 44,0 & 100,0 \\
\cline { 2 - 5 } & Percent & 58,5 & 41,5 & 100,0 \\
\hline \hline
\end{tabular}

Table 14: The Relations between the type of schools and question 1

\begin{tabular}{|c|c|c|c|c|c|}
\hline & & $\mathbf{A}$ & B & $\mathrm{C}$ & Total \\
\hline \multirow{2}{*}{ State } & Frequency & 3 & 8 & 33 & 44 \\
\hline & Percent & 7,0 & 18,0 & 75,0 & 100,0 \\
\hline \multirow{2}{*}{ Private } & Frequency & 0 & 0 & 2 & 2 \\
\hline & Percent & 0,0 & 0,0 & 100,0 & 100,0 \\
\hline \multirow{2}{*}{ Super } & Frequency & 0 & 0 & 4 & 4 \\
\hline & Percent & 0,0 & 0,0 & 100,0 & 100,0 \\
\hline \multirow{2}{*}{ Anatolian } & Frequency & 2 & 3 & 5 & 10 \\
\hline & Percent & 20,0 & 30,0 & 50,0 & 100,0 \\
\hline \multirow{2}{*}{$\begin{array}{l}\text { Technical and } \\
\text { Vacational }\end{array}$} & Frequency & 0 & 0 & 1 & 1 \\
\hline & Percent & 0,0 & 0,0 & 100,0 & 100,0 \\
\hline \multirow{2}{*}{ Science } & Frequency & 1 & 0 & 0 & 1 \\
\hline & Percent & 100,0 & 0,0 & 100,0 & 100,0 \\
\hline \multirow{2}{*}{ Total } & Frequency & 6 & 11 & 45 & 62 \\
\hline & Percent & 10,0 & 18,0 & 72,0 & 100,0 \\
\hline
\end{tabular}

Table 15: The Relations between the type of schools and question 2 


\begin{tabular}{|c|c|c|c|c|c|}
\hline & & Sometimes & Yes & No & Total \\
\hline \multirow[t]{2}{*}{ State } & $\begin{array}{l}\text { Frequenc } \\
\mathrm{y}\end{array}$ & 26 & 43 & 7 & 76 \\
\hline & Percent & 34,2 & 56,6 & 9,2 & 100,0 \\
\hline \multirow[t]{2}{*}{ Private } & $\begin{array}{l}\text { Frequenc } \\
\mathbf{y}\end{array}$ & 0 & 4 & 0 & 4 \\
\hline & Percent & 0,0 & 100,0 & 0,0 & 100,0 \\
\hline \multirow[t]{2}{*}{ Super } & $\begin{array}{l}\text { Frequenc } \\
\mathrm{y}\end{array}$ & 2 & 2 & 0 & 4 \\
\hline & Percent & 0,50 & 0,50 & 0 & 100,0 \\
\hline \multirow[t]{2}{*}{ Anatolian } & $\begin{array}{l}\text { Frequenc } \\
\mathrm{y}\end{array}$ & 5 & 10 & & 17 \\
\hline & Percent & 29,4 & 58,8 & 11,8 & 100,0 \\
\hline \multirow{2}{*}{$\begin{array}{l}\text { Technical } \\
\text { and } \\
\text { Vacational }\end{array}$} & $\begin{array}{l}\text { Frequenc } \\
\mathbf{y}\end{array}$ & 1 & 0 & 0 & 1 \\
\hline & Percent & 100,0 & 0,0 & 0,0 & 100,0 \\
\hline \multirow[t]{2}{*}{ Science } & $\begin{array}{l}\text { Frequenc } \\
\mathrm{y}\end{array}$ & 1 & 3 & 0 & 4 \\
\hline & Percent & 25,0 & 75,0 & 0,0 & 100,0 \\
\hline \multirow[t]{2}{*}{ Total } & $\begin{array}{l}\text { Frequenc } \\
\mathbf{y}\end{array}$ & 35 & 62 & 9 & 106 \\
\hline & Percent & 33,0 & 58,5 & 8,5 & 100,0 \\
\hline
\end{tabular}

Table 16: The relations between the type of schools and question 3

\begin{tabular}{||l|l|l|l|l||}
\hline \multirow{3}{*}{ State } & & Yes & No & Total \\
\hline \multirow{3}{*}{ Private } & Frequency & 14 & 62 & 76 \\
\cline { 2 - 5 } & Percent & 18,4 & 81,6 & 100,0 \\
\hline \multirow{3}{*}{ Super } & Frequency & 1 & 3 & 4 \\
\hline \multirow{3}{*}{ Anatolian } & Frequency & 3 & 1 & 4 \\
\cline { 2 - 5 } and & Percent & 0,75 & 0,25 & 100,0 \\
\cline { 2 - 5 } Vacational & Percent & 11,8 & 88,2 & 100,0 \\
\hline \multirow{3}{*}{ Science } & Frequency & 0 & 1 & 1 \\
\cline { 2 - 5 } & Percent & 0,0 & 100,0 & 100,0 \\
\hline \hline \multirow{3}{*}{ Total } & Frequency & 0 & 4 & 4 \\
\cline { 2 - 5 } & Percent & 0 & 100,0 & 100,0 \\
\hline \multirow{2}{*}{} & Frequency & 20 & 86 & 106 \\
\cline { 2 - 5 } & Percent & 18,9 & 81,1 & 100,0 \\
\hline
\end{tabular}

Table 18: The relations between the type of schools and question 5

\begin{tabular}{||l|l|l|l|l||}
\hline & & A & B & Total \\
\hline \multirow{2}{*}{ State } & Frequency & 51 & 25 & 76 \\
\cline { 2 - 5 } & Percent & 67,1 & 32,9 & 100,0 \\
\hline \multirow{2}{*}{ Private } & Frequency & 2 & 2 & 4 \\
\cline { 2 - 5 } & Percent & 0,50 & 0,50 & 100,0 \\
\hline \multirow{2}{*}{ Auper } & Frequency & 4 & 0 & 4 \\
\cline { 2 - 5 } & Percent & 100,0 & 0,0 & 100,0 \\
\hline \multirow{2}{*}{$\begin{array}{l}\text { Technical } \\
\text { Vacational }\end{array}$} & Frequency & 14 & 3 & 17 \\
\cline { 2 - 5 } & Percent & 82,4 & 17,6 & 100,0 \\
\cline { 2 - 5 } Science & Percent & 100,0 & 0,0 & 1 \\
\hline \hline \multirow{2}{*}{ Total } & Frequency & 3 & 1 & 4 \\
\cline { 2 - 5 } & Percent & 75,0 & 25,0 & 100,0 \\
\cline { 2 - 5 } & Frequency & 75 & 31 & 106 \\
\cline { 2 - 5 } & Percent & 70,8 & 29,2 & 100,0 \\
\hline \hline
\end{tabular}

Table 17: The relations between the type of schools and question 4

\begin{tabular}{||l|l|l|l|l||l||}
\hline \multirow{3}{*}{ State } & & A & B & C & Total \\
\hline \multirow{2}{*}{ Private } & Frequency & 25 & 17 & 34 & 76 \\
\cline { 2 - 6 } & Percent & 32,9 & 22,4 & 44,7 & 100,0 \\
\hline \multirow{3}{*}{ Super } & Prequency & 2 & 2 & 0 & 4 \\
\hline \multirow{2}{*}{ Anatolian } & Frequency & 2 & 2 & 0 & 4 \\
\cline { 2 - 6 } & Percent & 0,50 & 0,50 & 0,0 & 100,0 \\
\hline \multirow{2}{*}{ Technical and } & Frequency & 9 & 4 & 4 & 17 \\
\cline { 2 - 6 } & Percent & 52,9 & 23,5 & 23,5 & 100,0 \\
\hline \multirow{3}{*}{ Science } & Percent & 0,0 & 100,0 & 0,0 & 100,0 \\
\hline \hline \multirow{2}{*}{ Total } & Frequency & 3 & 0 & 1 & 4 \\
\cline { 2 - 6 } & Percent & 75,0 & 0,0 & 0,25 & 100,0 \\
\hline \multirow{2}{*}{ Frequency } & 41 & 26 & 39 & 106 \\
\cline { 2 - 6 } & Percent & 38,7 & 24,5 & 36,8 & 100,0 \\
\hline
\end{tabular}

Table19: The relations between the type of schools and question 6 


\begin{tabular}{||l|l|l|l||l||}
\hline & & Yes & No & Total \\
\hline \multirow{3}{*}{ Girls } & Frequency & 22 & 10 & 32 \\
\cline { 2 - 5 } & Percent & 68,8 & 31,2 & 100,0 \\
\hline \multirow{3}{*}{ Boys } & Frequency & 40 & 34 & 74 \\
\cline { 2 - 5 } & Percent & 54,1 & 45,9 & 100,0 \\
\hline \hline \multirow{2}{*}{ Total } & Frequency & 62 & 44 & 106 \\
\cline { 2 - 5 } & Percent & 58,5 & 41,5 & 100,0 \\
\hline
\end{tabular}

Table 20: The relationship between the gender and question 1.

\begin{tabular}{||l|l|l|l|l||l||}
\hline & & A & B & C & Total \\
\hline \multirow{3}{*}{ Girls } & Frequency & 1 & 5 & 16 & 22 \\
\cline { 2 - 5 } & Percent & 0,05 & 0,23 & 0,72 & 100,0 \\
\hline \multirow{3}{*}{ Boys } & Frequency & 5 & 6 & 29 & 40 \\
\cline { 2 - 5 } & Percent & 0,72 & 0,13 & 0,15 & 100,0 \\
\hline \hline \multirow{3}{*}{ Total } & Frequency & 6 & 11 & 45 & 62 \\
\cline { 2 - 5 } & Percent & 0,72 & 0,10 & 0,18 & 100,0 \\
\hline
\end{tabular}

Table 21: The relationship between the gender and question 2

\begin{tabular}{||l|l|l|l|l||l||}
\hline & & Sometimes & Yes & No & Total \\
\hline \multirow{3}{*}{ Girls } & Frequency & 10 & 19 & 3 & 22 \\
\cline { 2 - 6 } & Percent & 31,3 & 59,4 & 9,4 & 100,0 \\
\hline \multirow{3}{*}{ Boys } & Frequency & 25 & 43 & 6 & 74 \\
\cline { 2 - 6 } & Percent & 33,8 & 58,1 & 8,1 & 100,0 \\
\hline \hline \multirow{3}{*}{ Total } & Frequency & 35 & 62 & 9 & 106 \\
\cline { 2 - 6 } & Percent & 33,0 & 58,5 & 8,5 & 100,0 \\
\hline
\end{tabular}

Table 22: The relationship between the gender and question 3

\begin{tabular}{||l|l|l|l||l||}
\hline & & A & B & Total \\
\hline \multirow{3}{*}{ Girls } & Frequency & 22 & 10 & 32 \\
\cline { 2 - 5 } & Percent & 68,8 & 31,3 & 100,0 \\
\hline \multirow{3}{*}{ Boys } & Frequency & 53 & 21 & 74 \\
\cline { 2 - 5 } & Percent & 71,6 & 28,4 & 100,0 \\
\hline \hline \multirow{2}{*}{ Total } & Frequency & 75 & 31 & 106 \\
\cline { 2 - 5 } & Percent & 70,8 & 29,2 & 100,0 \\
\hline
\end{tabular}

Table 23: The relationship between the gender and question 4

\begin{tabular}{||l|l|l|l|l||}
\hline & & Yes & No & Total \\
\hline \multirow{3}{*}{ Girls } & Frequency & 7 & 25 & 32 \\
\cline { 2 - 5 } & Percent & 21,9 & 78,1 & 100,0 \\
\hline \multirow{3}{*}{ Boys } & Frequency & 13 & 61 & 74 \\
\cline { 2 - 5 } & Percent & 17,6 & 82,4 & 100,0 \\
\hline \hline \multirow{3}{*}{ Total } & Frequency & 20 & 86 & 106 \\
\cline { 2 - 5 } & Percent & 18,9 & 81,1 & 100,0 \\
\hline
\end{tabular}

Table 24: The relationship between the gender and question 5

\begin{tabular}{||l|l|l|l|l||l||}
\hline & & A & B & C & Total \\
\hline \multirow{3}{*}{ Girls } & Frequency & 13 & 7 & 12 & 32 \\
\cline { 2 - 6 } & Percent & 40,6 & 21,9 & 37,5 & 100,0 \\
\hline \multirow{3}{*}{ Boys } & Frequency & 28 & 19 & 27 & 74 \\
\cline { 2 - 6 } & Percent & 73,1 & 25,7 & 36,5 & 100,0 \\
\hline \hline \multirow{3}{*}{ Total } & Frequency & 41 & 26 & 39 & 106 \\
\cline { 2 - 6 } & Percent & 38,7 & 24,5 & 36,8 & 100,0 \\
\hline \hline
\end{tabular}

Table 25: The relationship between the gender and question 6 